\title{
Cost-Effectiveness Analysis of Allopurinol Versus Febuxostat in Chronic Gout Patients: A U.S. Payer Perspective
}

\author{
Pranav K. Gandhi, BPharm, MS, PhD; William M. Gentry, PharmD; Qinli Ma, PhD; \\ and Michael B. Bottorff, PharmD, FCCP, CLS
}

\begin{abstract}
BACKGROUND: Gout is a chronic inflammatory condition associated with poor urate metabolism. Xanthine oxidase inhibitors such as allopurinol and febuxostat are recommended to reduce uric acid levels and to prevent gout attacks in adult patients. Under budget-driven constraints, health care payers are faced with the broader challenge of assessing the economic value of these agents for formulary placement. However, the economic value of allopurinol versus febuxostat has not be assessed in patients with gout over a 5-year time period in the United States.
\end{abstract}

OBJECTIVE: To evaluate the cost-effectiveness of allopurinol versus febuxostat in adult patients with gout over a 5-year time period from a U.S. health care payer's perspective.

METHODS: A Markov model was developed to compare the total direct costs and success of serum uric acid (SUA) level reduction associated with allopurinol and febuxostat. Treatment success was defined as patient achievement of a sUA level $<6 \mathrm{mg} / \mathrm{dL}(0.36 \mathrm{mmol} / \mathrm{L})$ at 6 months. Event probabilities were based on published phase III randomized clinical trials and included long-term sequelae from open-label extension studies. A hypothetical cohort of 1,000 adult gout patients with sUA levels of $\geq 8 \mathrm{mg} / \mathrm{dL}$ $(0.48 \mathrm{mmol} / \mathrm{L})$ who had received either allopurinol $300 \mathrm{mg}$ or febuxostat $80 \mathrm{mg}$ at model entry transitioned among the 4 health states defined by treatment success, treatment failure and switch, treatment dropout, and death. The length of each Markov cycle was 6 months. Costs were gathered from the RED B00K, Medicare fee schedules, Healthcare Cost and Utilization Project's Nationwide Inpatient Sample, and for a limited number of inputs, expert consultation. Direct costs included treatment drug costs, costs for prophylaxis drugs, diagnostic laboratory tests, and the treatment and management of acute gout flare. Resource utilization was based on clinical evidence and expert consultation. All costs were inflated to 2014 U.S. dollars and were discounted at $3 \%$ in the base case. One-way sensitivity analysis and probabilistic sensitivity analyses (PSAs) were performed to assess the robustness of the results.

RESULTS: The total per patient cost incurred over 5 years was $\$ 50,295$ for febuxostat and $\$ 48,413$ for allopurinol, with an incremental total cost of $\$ 1,882$. The expected percentage of treatment success during the 5 -year period was 72 for febuxostat and $\mathbf{4 2}$ for allopurinol, resulting in an incremental percentage of treatment success of 30 . The estimated incremental cost-effectiveness ratio for febuxostat compared with allopurinol was $\$ 6,322$ per treatment success over a 5-year time period. The one-way sensitivity analysis indicated that the results were sensitive to probability of treatment success for allopurinol, probability of treatment dropouts for both allopurinol and febuxostat, and the probability of failure and switch to allopurinol. PSAs demonstrated that at a willingness-to-pay threshold of $\$ 50,000$ per treatment success, febuxostat was cost-effective compared with allopurinol.

CONCLUSIONS: Febuxostat was found to be a cost-effective option compared with allopurinol based on a U.S. payer perspective.

J Manag Care Pharm. 2015;21(2):165-75

Copyright $\odot 2015$, Academy of Managed Care Pharmacy. All rights reserved.

\section{What is already known about this subject}

Gout is a chronic inflammatory condition that affects an estimated 8 million people in the United States.

Xanthine oxidase inhibitors such as allopurinol or febuxostat are recommended as first-line pharmacologic urate-lowering therapies to reduce serum urate production

Previous economic models comparing allopurinol with febuxostat have typically used preference-based endpoints (i.e., qualityadjusted life-years) and have applied non-U.S. costs.

\section{What this study adds}

This is the first study that accounted for relevant direct health care costs and compared the cost-effectiveness of allopurinol with febuxostat over a 5-year time period in the United States

This economic model, constructed using data from published head-to-head clinical trial data, estimated an incremental costeffectiveness ratio of $\$ 6,322$ per treatment success.

Use of febuxostat appeared cost-effective compared with allopurinol, addressing the significance of management of serum uric acid goals in adult patients with gout.

G out is a chronic inflammatory condition characterized by poor urate metabolism. ${ }^{1-3}$ It is associated with precipitation of monosodium urate crystals in joints and soft tissues. ${ }^{1-3}$ The prevalence of gout has risen over the past 2 decades and now affects approximately 8 million people in the United States. ${ }^{4}$ Hyperuricemia, defined as serum uric acid (sUA) levels $\geq 6.8$ milligrams per deciliter $(\mathrm{mg} / \mathrm{dL}$ ), is a common biochemical irregularity associated with deposition of monosodium urate or uric acid crystals, predisposing clinical manifestations of gout. ${ }^{5,6}$ Studies have shown a positive correlation between maintaining a patient's sUA at $<6.8 \mathrm{mg} / \mathrm{dL}$ and preventing gout flares. ${ }^{7,8}$ Current guidelines from the American College of Rheumatology (ACR) recommend the use of pharmacologic urate-lowering therapy (ULT) for patients diagnosed with gouty arthritis that present with tophus, frequent attacks, and past urolithiasis. ${ }^{9}$ The first-line pharmacologic ULTs for chronic gout are xanthine oxidase inhibitors (XOIs) such as Zyloprim (allopurinol) or Uloric (febuxostat), which are used to achieve a target goal of serum urate levels less than $6 \mathrm{mg} / \mathrm{dL}$, with optimal levels below $5 \mathrm{mg} / \mathrm{dL} .^{9}$ Probalan (probenecid), an antihyperuricemic agent, is recommended as an 


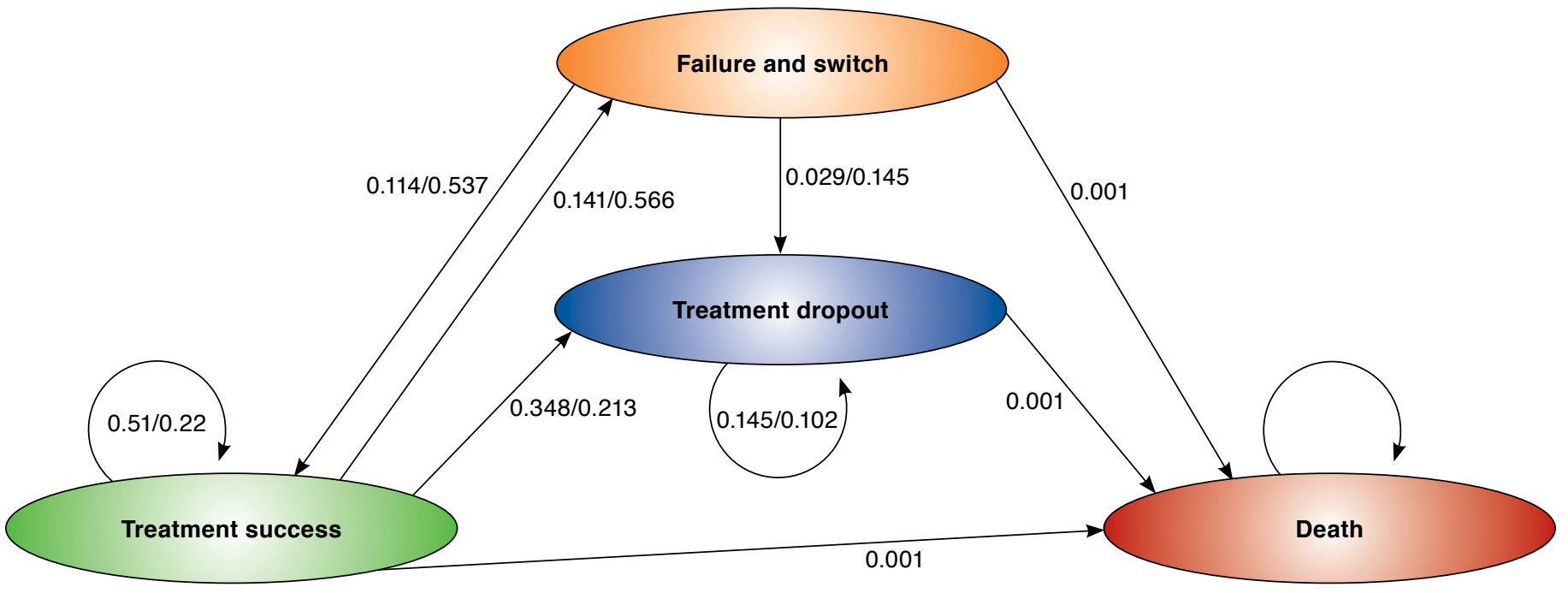

aTreatment success was defined when patients achieved sUA levels $<6 \mathrm{mg} / \mathrm{dL}(0.36 \mathrm{mmol} / \mathrm{L})$ at 6 months.

${ }^{b}$ Base-case probabilities for the first 6 months (for febuxostat/allopurinol) are presented.

$\mathrm{mg} / \mathrm{dL}=$ milligram per deciliter $; \mathrm{mmol} / \mathrm{L}=$ millimole per liter; $s U A=$ serum uric acid.

alternative first-line pharmacologic treatment option if at least $1 \mathrm{XOI}$ agent is contraindicated or not tolerated. The ACR guidelines recommend a combination of an oral ULT with an XOI agent and a uricosuric agent (e.g., probenecid) when the sUA target goals have not been achieved by appropriate XOI dosing. Pegloticase (Krystexxa, Savient Pharmaceuticals), a PEGlycated uric acid specific enzyme, is recommended as a third-line therapy to treat adult patients with chronic gout refractory to, or intolerant of, conventional oral XOIs and uricosuric agents. ${ }^{9}$ Probenecid and third-line pegloticase therapy were not included as comparative treatment options due to the lack of clinical trial data evaluating the long-term safety and efficacy of these therapies or consensus on how patients would be treated after failing 2 ULTs.

The recommended dose for allopurinol can be in the range of $100 \mathrm{mg}-800 \mathrm{mg}$ daily; however, it is most commonly dosed at $100 \mathrm{mg}-300 \mathrm{mg}$ daily. ${ }^{10,11}$ The recommended dose of febuxostat for treatment of hyperuricemia in patients with gout is $40 \mathrm{mg}$ or $80 \mathrm{mg}$ once daily. Febuxostat, a nonpurine selective XOI, was approved in 2009 by the U.S. Food and Drug Administration based upon phase II, phase III, and long-term, randomized, open-label extension studies for the treatment of hyperuricemia in patients with gout. ${ }^{6,12-15}$ Several studies have evaluated the cost-effectiveness of febuxostat compared with allopurinol in patients with gout where the findings support the use of febuxostat as a cost-effective strategy. ${ }^{16-20}$ However, some of these studies cannot be extrapolated to the U.S. set- ting due to non-U.S. costs, differential pricing strategies, treatment pattern data, and different regulatory systems. ${ }^{16-18}$ On the other hand, other studies either did not account for costs associated with health care resources typically reported in patients with gout or were evaluated only for a specific subgroup of gout patients (i.e., chronic kidney disease (CKD) stage $3 / 4$ gout patients with $s U A \geq 8 \mathrm{mg} / \mathrm{dL}$ ). ${ }^{19,20}$ To address this gap in knowledge and to fully understand the complete economic value over long-term sequelae, a Markov model was developed to compare the cost-effectiveness of allopurinol with febuxostat over a 5-year time period.

Data suggest that long-term treatment (sUA $<6 \mathrm{mg} / \mathrm{dL}$ ) of gout patients can lead to reduction in gout flares and resolution of tophi in the majority of patients. ${ }^{5}$ Cost-effectiveness studies have increasingly been used to demonstrate the economic appraisal of various treatment options based on the payer's perspective. Under budgetary constraints, health care payers are often faced with the broader challenge of assessing the economic value and making decisions regarding formulary placement of various treatment options. To determine whether the new treatment option offers potential benefits compared with the existing treatment options will typically be based upon its clinical relevance and economic value. The purpose of this study was to evaluate the cost-effectiveness of allopurinol versus febuxostat in patients with gout over a 5-year time period from a U.S. health care payer perspective. 
TABLE 1 Base-Case Undiscounted Probabilities and Ranges Used in One-Way Sensitivity Analyses

\begin{tabular}{|c|c|c|c|c|}
\hline Variable & $\begin{array}{c}\text { Febuxostat } 80 \mathrm{mg} \\
\text { Base-Case (Mean \%) }\end{array}$ & Range & $\begin{array}{l}\text { Allopurinol } 300 \mathrm{mg} \\
\text { Base-Case (Mean \%) }\end{array}$ & Range \\
\hline \multicolumn{5}{|c|}{ FACT, APEX, EXCEL, and FOCUS clinical trial studies, and Beard et al. ${ }^{17}:$ Patients achieved treatment success (sUA levels $<6 \mathrm{mg} / \mathrm{dL}$ ) } \\
\hline Cycle 1 & 51 & $46-56$ & 22 & $16.5-27.5$ \\
\hline Cycle 2 & 74 & - & 36 & - \\
\hline Cycle 3 & 88 & - & 81 & - \\
\hline Cycle 4 & 89 & - & 78.6 & - \\
\hline Cycle 5 & 89 & - & 73.3 & - \\
\hline Cycle 6 & 91 & - & 90 & - \\
\hline Annual average $^{\text {a }}$ (cycle 7-cycle 8) & 92 & - & 90 & - \\
\hline Annual average (cycle 9-cycle 10) & 93 & - & 89 & - \\
\hline \multicolumn{5}{|c|}{ FACT, APEX, and EXCEL clinical trial studies, and Beard et al. ${ }^{17}$ : Patients failed to achieve sUA levels and switched therapy } \\
\hline Cycle 1 & 14.1 & $9-19$ & 56.6 & $51-61$ \\
\hline Cycle 2 & 11.4 & - & 53.7 & - \\
\hline Cycle 3 & 5.8 & - & 10.7 & - \\
\hline Cycle 4 & 4.8 & - & 13.1 & - \\
\hline Cycle 5 & 7.5 & - & 21.1 & - \\
\hline Cycle 6 & 5.5 & - & 4.4 & - \\
\hline Annual averagea (cycle 7 -cycle 8) & 2.6 & - & 4.4 & - \\
\hline Annual average ${ }^{a}$ (cycle 9-cycle 10) & 5.1 & - & 7.4 & - \\
\hline \multicolumn{5}{|c|}{ FACT, APEX, and EXCEL clinical trial studies, and Beard et al. ${ }^{17}$ : Treatment dropout rates } \\
\hline Cycle 1 & 34.8 & $30-40$ & 21.3 & $16-26$ \\
\hline Cycle 2 & 14.5 & - & 10.2 & - \\
\hline Cycle 3 & 6.1 & - & 8.2 & - \\
\hline Cycle 4 & 6.1 & - & 8.2 & - \\
\hline Cycle 5 & 3.4 & - & 5.5 & - \\
\hline Cycle 6 & 3.4 & - & 5.5 & - \\
\hline Annual averagea (cycle 7 -cycle 8) & 5.2 & - & 5.5 & - \\
\hline Annual average ${ }^{\text {a }}$ (cycle 9-cycle 10) & 1.7 & - & 3.4 & - \\
\hline \multicolumn{5}{|c|}{ APEX and FACT studies, and Beard et al. ${ }^{17}$ : Number of acute gout flares, with 8 weeks of prophylaxis } \\
\hline $0-6$ months & 1.12 & & 0.92 & \\
\hline \multicolumn{5}{|c|}{ Probability of experiencing flare, after the first 6-month cycle, among those who have success or failure } \\
\hline Patients achieving treatment success (sUA levels $<6.0 \mathrm{mg} / \mathrm{dL}$ ) & 0.08 & & 0.11 & \\
\hline \multicolumn{5}{|c|}{$\begin{array}{l}\text { Note: Probabilities for those who achieved treatment success (sUA levels }<6 \mathrm{mg} / \mathrm{dL} \text { ) and for those who failed to achieve sUA levels and switched to other treatment optic } \\
\text { (i.e., allopurinol or febuxostat) for cycles 3-6 were taken from the EXCEL clinical study. } 5 \\
\text { aAdditional long-term probabilities were taken from the FOCUS clinical trial and Beard et al. } 17,21 \\
\text { APEX=Allopurinol-and Placebo-controlled Efficacy study of febuXostat; EXCEL=fEbuXostat/allopurinol Comparative Extension Long-term study; FACT=Febuxostat } \\
\text { versus Allopurinol Controlled Trial; FOCUS = Febuxostat Open-label Clinical Trial of Urate-Lowering Efficacy and Safety extension study; mg=milligram; } \\
m g / d L=\text { milligram per deciliter; sUA = serum uric acid. }\end{array}$} \\
\hline
\end{tabular}

\section{Methods}

A Markov model was designed to allow comparisons over a 5 -year time period. Clinical trial data comparing allopurinol with febuxostat were available over variable time periods (from 6 months up to 60 months). ${ }^{5,6,13,21}$ The first-year probabilities used in the economic model were pooled estimates based on information from 2 randomized controlled trials (RCTs) for allopurinol and febuxostat: the Febuxostat versus Allopurinol Control Trial (FACT; $\mathrm{n}=762$ ) observed over 52 weeks and the 28-week Allopurinol and Placebo-controlled Efficacy study of febuXostat (APEX) ${ }^{6,13}$ This approach was appropriate because the 2 clinical trials, FACT and APEX, used identical patient populations and efficacy and safety endpoints. Both RCTs com- pared febuxostat $80 \mathrm{mg} / 120 \mathrm{mg}$ with allopurinol $100 \mathrm{mg} / 300$ mg and placebo. ${ }^{6,13}$ The subjects who completed 1 of the 2 phase III RCTs (FACT or APEX) were enrolled in EXCEL, a long-term, randomized, open-label extension study $(\mathrm{n}=1,086)$, and monitored for up to 40 months of ULT. ${ }^{5}$ Subjects in the EXCEL clinical trial were assigned to fixed-dose daily ULT with febuxostat ( $80 \mathrm{mg}$ or $120 \mathrm{mg}$ ) or allopurinol (300 mg). The primary endpoint to evaluate the efficacy was the percentage of patients with serum urate concentration of $<6.0 \mathrm{mg} / \mathrm{dL}$ in all the 3 studies. ${ }^{5,6,13}$ Clinical trial evidence suggests that allopurinol doses of $300 \mathrm{mg}$ or less daily fail to achieve target sUA levels. ${ }^{9}$ However, allopurinol dosing of $300 \mathrm{mg}$ daily was compared with febuxostat $80 \mathrm{mg}$ for the following reasons: (a) lack of long-term safety and clinical trial data of doses greater 


\begin{tabular}{|c|c|c|c|}
\hline Resource Use & $\begin{array}{l}\text { Base-Case Value } \\
\text { Unit Costs (US\$) }\end{array}$ & Range & Data Source \\
\hline \multicolumn{4}{|l|}{ Costs of Therapy (6 months) } \\
\hline Febuxostat & $1,412.16$ & $1,059-1,765$ & RED BOOK 27 \\
\hline Allopurinol & 23.18 & $17.40-29.00$ & RED BOOK 27 \\
\hline Naproxen & 27.80 & $20.85-34.75$ & $\mathrm{RED} \mathrm{BOOK}^{27}$ \\
\hline \multicolumn{4}{|l|}{ Administration and monitoring } \\
\hline Outpatient physician visit & 108 per visit & $81.00-135.00$ & CPT code: 9921428 \\
\hline Inpatient physician visit & 72.36 per visit & $54.30-90.50$ & CPT code: $99232^{28}$ \\
\hline \multicolumn{4}{|l|}{ Laboratory tests } \\
\hline \multicolumn{4}{|l|}{ Febuxostat } \\
\hline Blood uric acid test (1 per year; 2 if goals not achieved) & 6.16 & - & HCPCS code: $84550^{28}$ \\
\hline Liver tests (ALT, AST; baseline, 2 months, 4 months, every year after first year) & 11.14 & - & HCPCS code: $80076^{28}$ \\
\hline Liver tests (alkaline phosphatase; baseline, 2 months, 4 months, every year after first year) & 9.96 & - & HCPCS code: $84078^{28}$ \\
\hline \multicolumn{4}{|l|}{ Allopurinol } \\
\hline Blood uric acid test (1 per year; 2 if goals not achieved) & 6.16 & - & HCPCS code: $84550^{28}$ \\
\hline Serum creatinine test (initiate then every year after first year) & 12.89 & - & HCPCS code: $82575^{28}$ \\
\hline Liver tests (ALT, AST; initiate then every year after first year) & 11.14 & - & HCPCS code: $80076^{28}$ \\
\hline Liver tests (alkaline phosphatase; initiate then every year after first year) & 9.96 & - & HCPCS code: $84078^{28}$ \\
\hline Complete blood count (initiate then every year after first year) & 10.61 & - & HCPCS code: $85025^{28}$ \\
\hline \multicolumn{4}{|l|}{ Other costs } \\
\hline Emergency department visits & 144 & $108-180$ & $\begin{array}{l}\text { Wu et al. }{ }^{1} \text { and expert } \\
\text { opinion }\end{array}$ \\
\hline Hospitalizations & 6,887 & $5,166-8,609$ & HCUP30 \\
\hline
\end{tabular}

$A L T=$ alanine aminotransferase; $A S T=$ aspartate aminotransferase; $C P T=$ Current Procedural Terminology; HCPCS = Healthcare Common Procedure Coding System; HCUP = Healthcare Cost and Utilization Project; $\$ U S=U . S$. dollars.

than $300 \mathrm{mg}$ of allopurinol ${ }^{9}$ and (b) suggestion of practice survey data that titration of allopurinol dosing of greater than 300 $\mathrm{mg}$ is seldom attempted by physicians. ${ }^{22}$

\section{Model Structure and Model Inputs}

The cost-effectiveness of allopurinol compared with febuxostat in treatment of patients with gout was evaluated using a Markov model (DATA, Treeage Software, Inc., Williamstown, MA). The Markov model consisted of 4 health states as presented in Figure 1 with each cycle length of 6 months. The 6-month cycle length was chosen because the time period was appropriate to determine treatment and clinical benefit and also represented the frequency of efficacy assessment in clinical trials. ${ }^{6,13}$ The model was used to consider a cohort of 1,000 adult gout patients (mean age: 43 years) with sUA levels of at least $8 \mathrm{mg} / \mathrm{dL}(0.48$ millimoles per liter $[\mathrm{mmol} / \mathrm{L}])$ and who had received allopurinol or febuxostat. Treatment success was defined as patient achievement of a sUA level $<6 \mathrm{mg} / \mathrm{dL}$ $(0.36 \mathrm{mmol} / \mathrm{L})$ at 6 months. After each defined 6 -month cycle, depending on the transition probabilities, patients with treatment success could remain in the specified health state (sUA level $<6 \mathrm{mg} / \mathrm{dL}, 0.36 \mathrm{mmol} / \mathrm{L}$ ) and were expected to continue treatment; those with sUA levels $\geq 6 \mathrm{mg} / \mathrm{dL}$ experienced treatment failure, whereas the remaining portion experienced treat- ment dropouts or death. Treatment-specific acute gout flare rates for the first 6 months were based on the pooled estimates from the APEX and FACT clinical trial data. ${ }^{6,13,17}$ It is a wellknown paradoxical fact that administering ULT leads to acute gout flares. ${ }^{17}$ Clinical trial data suggest higher acute gout flare rates associated with febuxostat compared with allopurinol in the initial treatment periods, primarily due to greater reduction in sUA levels with febuxostat (gout flare rates are presented in Table 1). ${ }^{17}$ The EXCEL clinical trial and another published study provided additional long-term data on acute gout flare rates. ${ }^{5,17}$ Patients developing acute gout flares during treatment were managed by nonsteroidal anti-inflammatory drugs (NSAIDs) or colchicine for a period of 8 weeks per 6-month cycle. ${ }^{17}$

With failure to achieve treatment success (sUA $\geq 6 \mathrm{mg} / \mathrm{dL}$ ), patients switched to the other treatment option (i.e., allopurinol or febuxostat). This approach was chosen because the failure of model patients to switch therapy can influence real-life settings, which in turn can underestimate the clinical benefit, costs, and long-term safety in these patients. The clinical trial data that were used in this study do not reflect "realworld" discontinuation after treatment failures. In the Markov model, a few criteria were defined for stopping the treatment option. First, it was assumed that patients with 2 consecutive 
treatment failures would discontinue treatment and would remain in that state for rest of the study period. Second, treatment dropouts and discontinuation rates were applied in this model given that poor adherence to ULT is a common problem in gout patients. ${ }^{9,23}$ The transition probabilities of maintaining treatment success, failure, and treatment dropout over the first year were derived from the APEX and FACT clinical trials (Table 1). ${ }^{6,13}$ In the absence of long-term data after 52 weeks, the transition probabilities of maintaining long-term response, failure, and treatment dropouts for 13-36 months were taken from the EXCEL clinical study. ${ }^{5}$ Additional long-term data for urate-lowering and clinical efficacy and safety were obtained from a previously published study and the 5-year Febuxostat Open-label Clinical Trial of Urate-Lowering Efficacy and Safety (FOCUS) extension study. ${ }^{17,21}$ Since the mortality rates across both treatment arms did not significantly differ, it was assumed that the transition probabilities to death were identical for both treatment arms. ${ }^{24}$

The model was designed from the health care payer perspective in the United States. Direct medical costs and effectiveness measures were estimated over a 5 -year time period. This time period was chosen for 3 reasons: (1) this was the closest approximation to the long-term EXCEL and FOCUS clinical studies ${ }^{5,21}$; (2) long-term sequelae for patients with gout can be presented well with a 5-year study period; and (3) U.S. health care payers typically prefer a shorter time period for economic evaluations. ${ }^{25,26}$ Cost-effectiveness was expressed as cost per treatment success where success was defined as an sUA level reduction of $<6 \mathrm{mg} / \mathrm{dL}$. A discount rate of $3 \%$ was applied for both cost and effectiveness in the base-case analysis. All costs were presented in 2014 U.S. dollars.

\section{Data Sources and Assumptions for Resource Use and Cost Estimates}

Since this study was conducted from a U.S. health care payer perspective, only direct costs were estimated, which included treatment drug costs and medical costs, including costs for prophylaxis drugs, diagnostic laboratory tests, and those associated with treatment and management of acute gout flare. Monthly cost of therapy included the cost of the treatment drug (allopurinol or febuxostat) based on annual wholesale acquisition cost (WAC) ${ }^{27}$ Consistent with treatment dose from the phase III clinical trials, ${ }^{6,13}$ the WAC for allopurinol $300 \mathrm{mg}$ and febuxostat $80 \mathrm{mg}$ were calculated for this study (Table 2). Unit costs associated with laboratory monitoring tests were based on the 2014 Medicare fee schedule. ${ }^{28}$ Outpatient physician (Current Procedural Terminology [CPT] code 99214) and inpatient physician visits (CPT code 99232) were based on the Medicare physician fee schedule..$^{28}$ The costs associated with hospitalizations were obtained from 2012 Healthcare Cost and Utilization Project (HCUP) statistics from the HCUP-3 Nationwide Inpatient Sample for Principal Diagnoses for gout and adjusted for inflation to 2014 U.S. dollars using the medical care component of the Consumer Price Index (CPI). 29,30

Costs associated with emergency department (ED) visits for patients with gout have not been reported clearly. ED estimates were derived based on a 2012 study by Wu et al. (2012) and expert consultation. Since the estimates obtained from both of these sources were highly skewed and differed based on sample size, weighted estimates were calculated. The total charge for ED visits based on these sources was adjusted using a cost-tocharge ratio (CCR) to convert into actual costs. The Agency for Healthcare Research and Quality recommends that hospitalwide CCRs should be multiplied by appropriate adjustment factors (AFs) for the patient's Medicare Severity Diagnosis Related Groups or Clinical Classification Software (CCS) category to obtain a more accurate CCR, which in turn can provide a more accurate cost estimate. ${ }^{31}$ We multiplied the CCRs with AFs for the principal CCS category for gout and other crystal arthropathies to obtain the most accurate CCR (0.63326). The calculated costs were inflated to 2014 U.S. dollars using the medical care component of the CPI, since the charges and CCR estimate were based on 2009 values. Costs of adverse events were not incorporated in this study because the adverse events rates (including cardiovascular adverse event rates) did not differ significantly across the 2 treatment groups. ${ }^{6,13}$

The recommendations and assumptions for the type and frequency of monitoring units were based on clinical evidence and consultation with a pharmacist with expertise and knowledge about patients with gout (the pharmacist was a colleague of the authors of this study). One-time costs associated with blood uric acid tests were added for both drugs, and specific laboratory costs were added for patients treated with allopurinol (Table 2). Anti-inflammatory agents such as oral colchicine (0.5 mg or $0.6 \mathrm{mg}$ orally once or twice a day), low-dose NSAIDs (e.g., naproxen $250 \mathrm{mg}$ orally twice a day), or low-dosage prednisone or prednisolone (defined as $\leq 10 \mathrm{mg}$ /day) are also recommended for gout attack prophylaxis. ${ }^{9,10}$ In addition to the drug costs, the costs for the treatment and management of acute gout flare were assumed to include the costs for drug prophylaxis for 8 weeks (naproxen 250 mg orally twice a day) and 1 outpatient visit. It was assumed that patients with gout flare would incur drug costs, drug prophylaxis costs for 8 weeks (naproxen $250 \mathrm{mg}$ orally twice a day), 2 outpatient visits, and 1 ED visit for a 6-month cycle. It was also assumed that patients with treatment dropouts would incur 2 outpatient visits, 2 ED visits over a 6-month period, and 1 inpatient visit and 1 hospitalization every year for the remainder of the study period. Table 2 presents resource use and health care costs associated with allopurinol and febuxostat.

The cost-effectiveness analysis model included costs and effectiveness outcomes for each treatment drug, incremental costs, incremental effectiveness, and incremental cost-effectiveness ratios (ICER) comparing allopurinol with febuxostat. 
TABLE 3 Cost-Effectiveness Results for the Base Case

\begin{tabular}{|c|c|c|c|c|c|}
\hline & Total Costs (\$US)a & Total Effectiveness (\%) ${ }^{b}$ & $\begin{array}{c}\text { Incremental Total Costs } \\
\text { (\$US)c }\end{array}$ & $\begin{array}{l}\text { Incremental Total } \\
\text { Effectiveness }{ }^{\mathrm{b}, \mathrm{c}}\end{array}$ & ICER $^{\mathrm{d}}$ \\
\hline Allopurinol & 48,413 & 42 & & & \\
\hline Febuxostat & 50,295 & 72 & 1,882 & 30 & 6,322 \\
\hline \multicolumn{6}{|c|}{$\begin{array}{l}{ }^{a} \text { Costs are in year } 2014 \text { values. } \\
b \text { Percentage of treatment success. } \\
\text { cCosts and effectiveness were discounted at } 3 \% . \\
\text { dDue to rounding, the ICER value may not add up exactly the same. } \\
\text { ICER=incremental cost-effectiveness ratio; } \$ U S=U . S \text {. dollars. }\end{array}$} \\
\hline
\end{tabular}

\section{Sensitivity Analyses}

One-way sensitivity analyses and multivariate probabilistic sensitivity analyses (PSAs) were conducted using a Monte Carlo simulation to examine the influence of variations in several key model inputs and assumptions on the model results. For the one-way sensitivity analysis, costs associated with treatment, hospitalization, outpatient and inpatient physician visits, and ED visits were varied by $\pm 25 \%$ of the base value to evaluate the sensitivity of these assumptions on cost-effectiveness results. Base-case probabilities for treatment success and treatment failure were also varied using one-way sensitivity analyses (Table 1). Due to differences in the discontinuation rates in clinical trials compared with a real-world setting, the base-case probability for treatment dropouts was also varied in one-way sensitivity analysis. Since the frequency of resources associated with treatment dropouts was based on clinical judgment and expert consultation, the frequency of resources were varied to evaluate its impact on the robustness of the analysis. In a secondary analysis, it was assumed that treatment dropouts would incur 2 outpatient visits and 1 ED visit over a 6-month period and 1 inpatient visit every year for the remainder of the study period.

For the multivariate PSAs using a Monte Carlo simulation, all cost parameters and base-case probabilities were simultaneously varied 10,000 times to evaluate the robustness of the results in the model. Beta distributions bounded by 0 and 1 were used for probability parameters where the means for these distributions were set equal to their base-case values, and the standard deviations (SDs) were approximated by $\pm 0.05 \mu$, where $\mu$ was the base-case mean value of the parameter. Gamma distributions were applied to represent the uncertainty in the cost parameters where the means for these distributions were set equal to their base-case values, and the SDs were approximated by $\pm 25 \%$ of the base-case mean value of the parameter. This approximation was made because the relevant variance data were not available in the sources for the cost parameters. The distributions of ICERs at 5-year intervals were plotted in cost-effectiveness acceptability curves (CEACs). Different willingness-to-pay (WTP) thresholds were derived from the CEACs to determine the cost-effectiveness of febuxostat compared with allopurinol.

\section{Results}

\section{Base-Case Analysis}

The per patient total costs incurred over a 5-year time period were estimated at $\$ 48,413$ for allopurinol and $\$ 50,295$ for febuxostat, resulting in a 5-year incremental total cost of $\$ 1,882$ for febuxostat compared with allopurinol. The estimated effectiveness based on percentage of treatment success during the 5-year time period was 42 and 72 for allopurinol and febuxostat, respectively. This resulted in an incremental percentage of treatment success of 30, comparing febuxostat with allopurinol. Thus, the incremental total costs per treatment success with the use of febuxostat compared with allopurinol were $\$ 6,322$ over 5 years (Table 3 ).

\section{Sensitivity Analysis}

One-way sensitivity analyses demonstrated that ICER results were impacted by several key parameters; study findings were most sensitive to the probabilities in the model (Figure 2). The results for one-way sensitivity analyses ranged from a lower limit of $-\$ 4,863$ per treatment success to an upper limit of $\$ 18,936$ per treatment success when comparing febuxostat with allopurinol. The lower limit and upper limit were obtained by varying the probability of treatment dropouts for allopurinol (from 0.16 to 0.26 ). The 4 most sensitive input parameters included probabilities of treatment success for allopurinol, treatment dropout for allopurinol, treatment dropout for febuxostat, and failure and switch to allopurinol. Febuxostat was the dominant strategy compared with allopurinol when decreasing the probability of treatment success for allopurinol (from 0.22 to 0.165 ), increasing the probability of treatment dropouts for allopurinol (from 0.21 to 0.26 ) or the probability of failure of febuxostat and switch to allopurinol (from 0.14 to 0.19), or decreasing the probability of treatment dropouts for febuxostat (from 0.35 to 0.30 ). The secondary analysis results when decreasing the frequency of resources associated with treatment dropouts demonstrated that the ICER with febuxostat versus the ICER with allopurinol was $\$ 14,940$ over a 5 -year time period (data not presented). Variations in other input parameters led to marginal changes in 5-year ICER values. 


\section{FIGURE 2 Tornado Diagram of One-Way Sensitivity Analyses Showing} Influence of Base-Case Costs and Probabilities

\section{ICER Ranges for One-Way Sensitivity Analyses, Febuxostat versus Allopurinol}

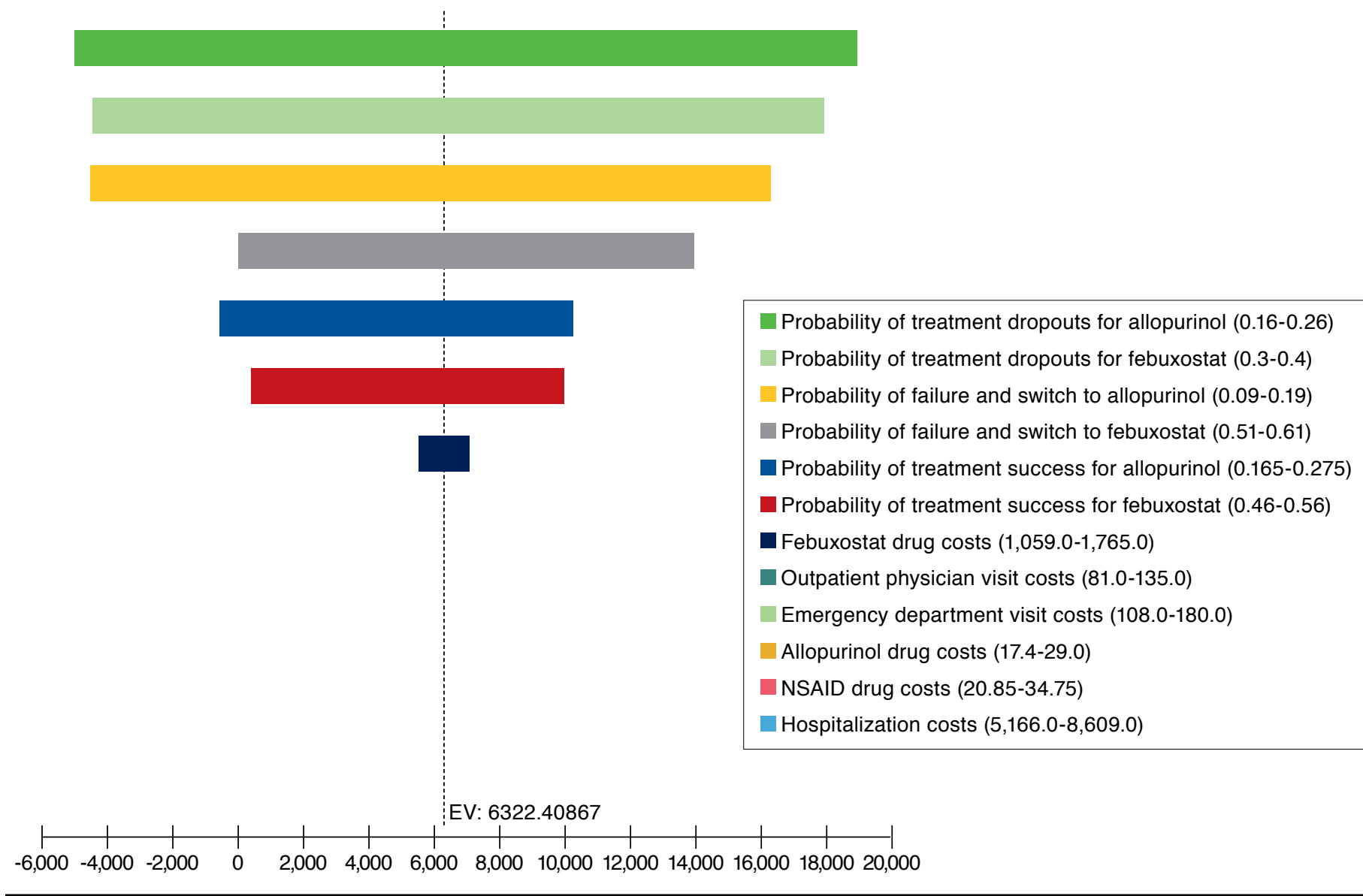

${ }^{a}$ Cost parameters were varied $\pm 25 \%$ around the base value.

$E V=$ expected value ICER = incremental cost-effectiveness ratio; NSAID = nonsteroidal anti-inflammatory drug

PSAs performed 10,000 iterations of the model, which demonstrated that febuxostat had a $51.5 \%$ chance of being costeffective at a WTP threshold of $\$ 10,000$ per treatment success (Figure 3) and a 65\% chance of being cost-effective at a WTP threshold of $\$ 50,000$ per treatment success.

\section{Discussion}

The Markov model in this study demonstrated that using febuxostat is cost-effective compared with allopurinol in the United States, with an ICER of $\$ 6,322$ per treatment success over a 5 -year time period. The one-way sensitivity analyses indicated that the results were most sensitive to the probabilities of treatment success for allopurinol, treatment dropouts for allopurinol, treatment dropouts for febuxostat, and the probability of failure and switch to allopurinol. Surprisingly, none of the cost parameters significantly influenced the ICER values, especially since some of the cost values and frequency of resource consumption were based on expert consultation. Nonetheless, it is worth mentioning that although assumptions were varied in the one-way sensitivity analyses, febuxostat was found to be a cost-effective option compared with allopurinol for adult patients with gout in the United States. PSAs showed that febuxostat had a greater than $51.5 \%$ probability of being cost-effective compared with allopurinol for a WTP threshold of at least $\$ 10,000$ per treatment success.

To our knowledge, this is the only study that has accounted for all relevant direct health care costs for allopurinol and febuxostat in patients with gout in the United States. Several other studies have used the ICER metric to assess the costeffectiveness of allopurinol versus febuxostat. ${ }^{16-20}$ As a part of 


\section{FIGURE 3 Cost-Effectiveness Acceptability Curve: Febuxostat Versus} Allopurinol for Treatment of Adult Gout Patients ${ }^{\mathrm{a}}$

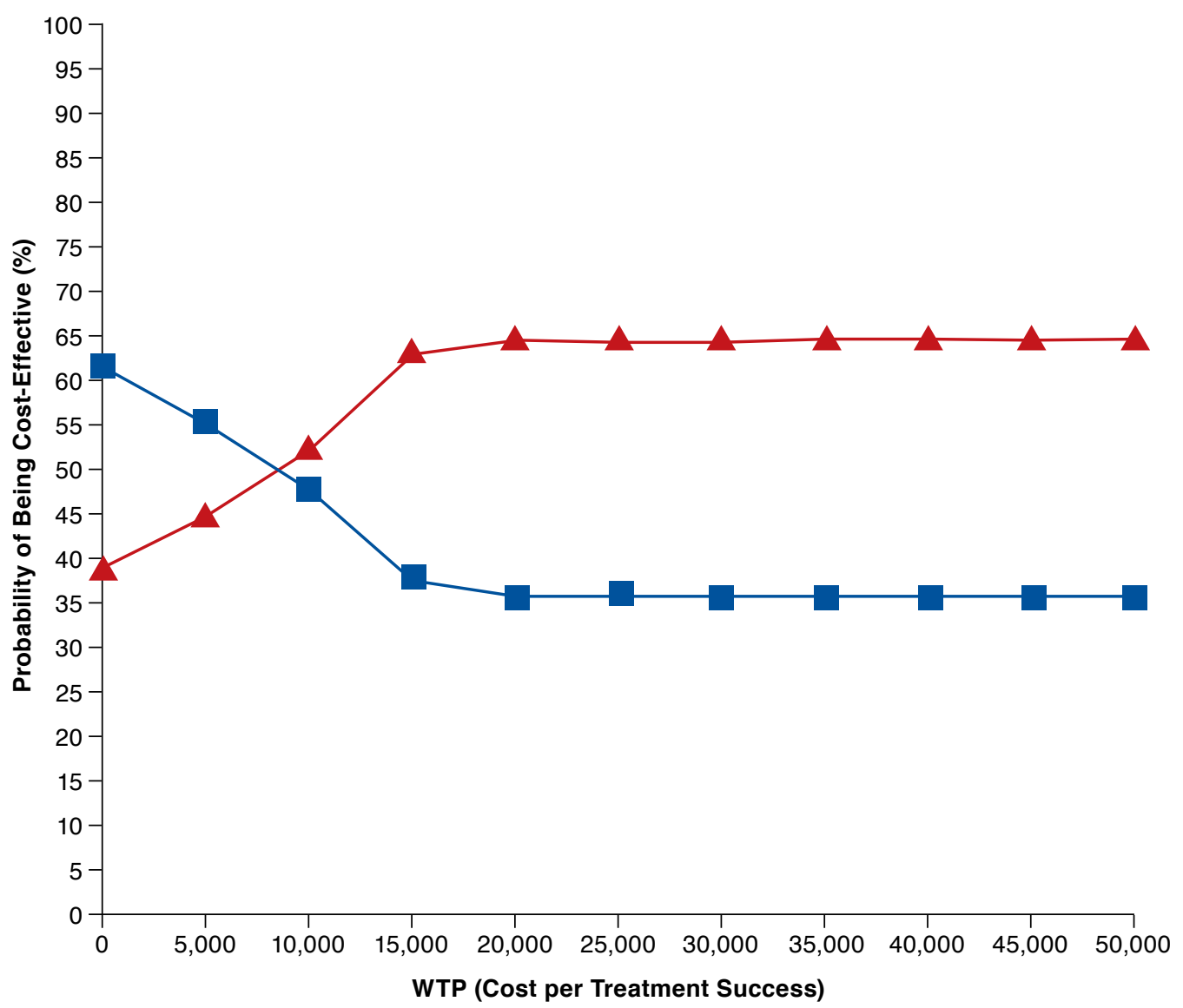

a Figure shows the probability of allopurinol and febuxostat being cost-effective at various WTP thresholds in 10,000 Monte-Carlo simulations. WTP= willingness to pay.

its submission to the National Institute for Health and Clinical Excellence in the United Kingdom, the drug manufacturer conducted an economic analysis comparing febuxostat at doses of $80 \mathrm{mg} /$ day or $120 \mathrm{mg} /$ day and allopurinol at $300 \mathrm{mg} /$ day over 1 - and 2-year time periods. The results showed an ICER of $£ 16,574$ (\$US25,920) and $£ 15,565$ (\$US24,342), respectively. ${ }^{16}$ However, it was argued that the results were based on imprecise estimates and biases and should be interpreted with caution. ${ }^{17}$ A recent economic study evaluated the cost-effectiveness of febuxostat compared with allopurinol in gout patients based on the perspectives of the National Health Service and the Social Services in Scotland. Febuxostat was found to be costeffective as a second-line therapy for treatment of patients with chronic hyperuricemia. An ICER of $£ 3,578$ /quality-adjusted life-year (QALY) was reported for second-line therapy with febuxostat $80 \mathrm{mg} / 120 \mathrm{mg}$ compared with allopurinol over a 5-year time period. ${ }^{17}$ In a separate study, a Markov model was conducted to compare the cost-effectiveness of febuxostat with allopurinol based on a Canadian public payer perspective. This economic study estimated an ICER of \$18,395/QALY for febuxostat versus allopurinol in patients with normal renal function. ${ }^{18}$ However, the numbers from these studies are not directly comparable to our study due to different health care systems, non-U.S. costs, cross-country cost variations, and use of preference-based endpoints (i.e., QALYs).

A study by Meltzer et al. (2012) found that a significant reduction in febuxostat drug cost would be required for it to dominate allopurinol. ${ }^{19}$ Although, the authors found febuxostat to be a cost-effective option, they did not account for relevant health care resource costs typically associated with gout patients. For instance, febuxostat incurs additional costs for liver function test abnormalities and the expected cost for 
gout flare prophylaxis. Moreover, the authors developed a simple decision tree model over a l-year time period and did not account for the continuous risk and change in health states over time in gout patients. A more recent study by Smolen et al. (2014) evaluated the cost-effectiveness of febuxostat versus allopurinol in CKD stage $3 / 4$ gout patients with sUA $\geq 8 \mathrm{mg} / \mathrm{dL}$ over a 5 -year time period. ${ }^{20}$ Although this study found fewer gout flares, fewer CKD stage 5 patients with gout, and reduced overall costs, the authors accounted for a specific subgroup of gout patients, thereby reducing the applicability of their study findings. Given that the economic evidence comparing allopurinol and febuxostat in the United States is limited, the present study provides timely information to payers regarding the economic value of using febuxostat compared with allopurinol in the United States.

Although the Markov model cannot represent the actual disease course in a real-world setting, it is a commonly used approach to estimate the costs and benefits, when the risk is continuous and there is a possibility that the health states may occur more than once. ${ }^{26,32}$ Data from the long-term extension study demonstrated that maintenance of sUA goals will lead to virtual elimination of acute gout flares and resolution of tophi status. ${ }^{5}$ These data demonstrate the significance of long-term management of sUA target goals that can lead to prevention and near elimination of acute gout flares and resolution of tophus status over time. Utilities were not considered in this study, since at the time of the study, there was no published evidence of utility values among gout patients in the United States. Prior evidence has reported the influence of long-term progression of gout on negative well-being and health-related quality of life among gout patients. ${ }^{17,33}$

\section{Limitations}

The recommendations and assumptions for the type and frequency of resource consumptions were based on clinical evidence and expert consultation. In addition, transition probabilities were derived from published clinical trial data and open label extension studies., ${ }^{5,6,13,21}$ Although the use of different sources for costs and probabilities is a common approach in cost-effectiveness studies, the varied assumptions can potentially influence the model results. However, one-way sensitivity analyses demonstrated that at certain values within the ranges for input parameters of probabilities of treatment success of allopurinol, treatment dropouts for allopurinol, treatment dropouts for febuxostat, and failure and switch to allopurinol, febuxostat was found to be a dominant treatment option compared with allopurinol (Figure 2). Another major assumption in this study was that patients discontinued treatment if they had 2 consecutive treatment failures. Although not all patients follow this treatment pattern in a real-world setting, current literature does not provide any direct evidence of these parameters. Moreover, as shown in clinical trials, most patients achieve sUA target goals within 12 months of therapy. ${ }^{6,13}$

We did not consider second- and third-line treatment options among patients with gout in the present study, since existing literature and publicly available data provide very limited direct evidence to demonstrate the influence of these parameters. It was also important that our study findings reflect the differences among the 2 treatment options of interest and not be confounded by effects from subsequent treatment options. The present study might be cautiously generalized to the real-world setting, since effectiveness parameters can be considerably different, such as treatment effectiveness, patient compliance, discontinuation rates, and presence of comorbid conditions. We chose not to incorporate costs associated with adverse events in the model estimation with use of either therapy. Clinical trial data suggest that adverse event rates among patients using either therapy were not found to be statistically different.

Finally, this study was conducted from a U.S. health care payer perspective and did not include indirect and societal cost estimates. Future studies should evaluate the cost-effectiveness of febuxostat versus allopurinol from a societal perspective to assess whether indirect and intangible costs influence the ICER values in any considerable way. The association between gout and significantly greater work absenteeism and negative impact on productivity than their counterparts without gout has previously been demonstrated ${ }^{34}$ It is worth noting that if the magnitude of indirect costs is correlated with the reduction in frequency of acute gout flares and resolution or reduction in tophus status over time, it is conceivable that the study findings may remain the same from a societal perspective.

\section{Conclusions}

This economic assessment is the first study to compare the cost-effectiveness of allopurinol with febuxostat among gout patients in the United States over a 5-year time period. The base-case analysis showed that the incremental costs per treatment success for febuxostat were $\$ 6,322$ over a 5-year period, suggesting that febuxostat may be a cost-effective option compared with allopurinol among adult patients with gout. The results were sensitive to base-case probabilities where we found that febuxostat was a dominant treatment option compared with allopurinol. These study findings can better inform health care payers regarding formulary coverage of treatment options used in adult patients with gout in the United States. 


\section{Authors}

PRANAV K. GANDHI, BPharm, MS, PhD, is Assistant Professor; WILLIAM M. GENTRY, PharmD, is Assistant Professor and Executive Associate Dean; and MICHAEL B. BOTTORFF, PharmD, FCCP, CLS, is Professor and Chair, Department of Pharmacy Practice, South College School of Pharmacy, Knoxville, Tennessee. QINLI MA, PhD, is Research Analyst, HealthCore, Wilmington, Delaware.

AUTHOR CORRESPONDENCE: Pranav K. Gandhi, BPharm, MS PhD, Assistant Professor, Department of Pharmacy Practice, South College School of Pharmacy, 400 Goodys Ln., Knoxville, TN 37922. E-mail:pgandhi@southcollegetn.edu.

\section{DISCLOSURES}

The authors have no direct or indirect financial relationships to declare. Study concept and design were contributed by Gandhi, Gentry, and Ma. Data were collected primarily by Gandhi, with assistance from Gentry, and interpreted by Gandhi. The manuscript was written primarily by Gandhi, assisted by Gentry and Ma. All authors contributed equally to manuscript revision.

\section{ACKNOWLEDGMENTS}

The authors would like to thank Dr. Jonathan Newsome for his expert consultation regarding the frequency of health care resources in gout patients.

\section{REFERENCES}

1. Wu EQ, Forsythe A, Guerin A, Yu AP, Latremouille-Viau D, Tsaneva M. Comorbidity burden, healthcare resource utilization, and costs in chronic gout patients refractory to conventional urate-lowering therapy. Am J Ther. 2012;19(6):e157-66.

2. Park H, Rascati KL, Prasla K, McBayne T. Evaluation of health care costs and utilization patterns for patients with gout. Clin Ther. 2012;34(3):640-52.

3. Li C, Martin BC, Cummins DF, Andrews LM, Frech-Tamas F, Yadao AM. Ambulatory resource utilization and cost for gout in United States. Am J Pharm Benefits. 2013;5(2):e46-e54.

4. Zhu Y, Pandya BJ, Choi HK. Prevalence of gout and hyperuricemia in the US general population: the National Health and Nutrition Examination Survey 2007-2008. Arthritis Rheum. 2011;63(10):3136-41.

5. Becker MA, Schumacher HR, MacDonald PA, Lloyd E, Lademacher C. Clinical efficacy and safety of successful longterm urate lowering with febuxostat or allopurinol in subjects with gout. J Rheumatol. 2009;36(6):1273-82.

6. Becker MA, Schumacher HR, Wortmann RL, et al. Febuxostat compared with allopurinol in patients with hyperuricemia and gout. N Engl J Med. 2005;353(23):2450-61

7. Halpern R, Fuldeore MJ, Mody RR, Patel PA, Mikuls TR. The effect of serum urate on gout flares and their associated costs: an administrative claims analysis. J Clin Rheumatol. 2009;15(1):3-7.

8. Wu EQ, Patel PA, Mody RR, et al. Frequency, risk, and cost of goutrelated episodes among the elderly: does serum uric acid level matter? J Rheumatol. 2009;36(5):1032-40.

9. Khanna D, Fitzgerald JD, Khanna PP, et al. 2012 American College of Rheumatology guidelines for management of gout. Part I: systematic nonpharmacologic and pharmacologic therapeutic approaches to hyperuricemia. Arthritis Care Res (Hoboken). 2012;64(10):1431-46.
10. Zhang W, Doherty M, Bardin T, et al. EULAR evidence based recommendations for gout. Part II: management. Report of a task force of the EULAR Standing Committee for International Clinical Studies Including Therapeutics (ESCISIT). Ann Rheum Dis. 2006;65(10):1312-24.

11. Allopurinol product information, scored tablets. Apotex Corp. May 2006. Available at: https://www.apotex.com/us/en/products/downloads/pil/ allo_imtb_ins.pdf. Accessed January 14, 2015.

12. Uloric (febuxostat) tablet for oral use. Takeda Pharmaceuticals America March 2013. Available at: http://general.takedapharm.com/content/file.asp $\mathrm{x}$ ?filetypecode=ULORICPI\&cacheRandomizer $=2 \mathrm{ce} 0 \mathrm{a} 084$-bab8-437c-9bf3ea20ef8f33a5. Accessed January 14, 2015

13. Schumacher HR Jr, Becker MA, Wortmann RL, et al. Effects of febuxostat versus allopurinol and placebo in reducing serum urate in subjects with hyperuricemia and gout: a 28-week, phase III, randomized, doubleblind, parallel-group trial. Arthritis Rheum. 2008;59(11):1540-48

14. Wortmann RL, Becker MA, Schumacher HR. Effect of febuxostat or allopurinol on the clinical manifestations of gout: reduction in gout flares and tophus size over time in the EXCEL trial. Poster presented at: American College of Rheumatology/Association of Rheumatology Health Professionals (ACR/ARHP) Annual Scientific Meeting; November 10-15, 2006; Washington, DC. [Abstract no. 1592].

15. Becker M, Schumacher H, MacDonald PA. Urate-lowering therapy in subjects with gout: interim results from the febuxostat comparative extension long-term study (EXCEL). Poster presented at: Annual European Congress of Rheumatology (EULAR); June 13-16, 2007; Barcelona, Spain. [Abstract no. THU0339].

16. Ipsen UK. Febuxostat for the treatment of gout: single technology appraisal submission to the National Institute for Health and Clinical Excellence. 2008. Available at: http://www.nice.org.uk/guidance/tal64/documents/manufacturer-ipsen-submission2. Accessed January 2015.

17. Beard SM, von Scheele BG, Nuki G, Pearson IV. Cost-effectiveness of febuxostat in chronic gout. Eur J Health Econ. 2014;15(5):453-63.

18. Redding L, Hornberger J, Cowens W, Chien R, Wang M. Cost-effectiveness of febuxostat in managing hyperuricemia in gout patients in Canada. Poster presented at: Canadian Agency for Drugs and Technologies in Health Symposium; April 3-5, 2011; Vancouver, British Columbia, Canada. Available at: http://www.cadth.ca/en/events/cadth-symposium-archives/2011-cadthsymposium/poster-presentations. Accessed January 9, 2015.

19. Meltzer M, Pizzi LT, Jutkowitz E. Payer decision-making with limited comparative and cost-effectiveness data: the case of new pharmacological treatments for gout. Evid Based Med. 2012;17(4):105-08.

20. Smolen LJ, Wielage RC, Gahn JC, Mitri G, Shiozawa A. The budgetary impact of febuxostat in the management of patients with gout. J Manag Care Pharm. 2014;20(Suppl 4-a):S52. [Abstract]. Available at: http://www.amcp. org/WorkArea/DownloadAsset. aspx?id=17840.

21. Schumacher HR Jr, Becker MA, Lloyd E, MacDonald PA, Lademacher C. Febuxostat in the treatment of gout: 5-yr findings of the FOCUS efficacy and safety study. Rheumatology (Oxford). 2009;48(2):188-94.

22. El-Zawawy H, Mandell BF. Managing gout: how is it different in patients with chronic kidney disease? Cleve Clin J Med. 2010;77(12):919-28.

23. Harrold LR, Andrade SE, Briesacher BA, et al. Adherence with urate-lowering therapies for the treatment of gout. Arthritis Res Ther. 2009;11(2):R46.

24. Arias E. United States life tables, 2006. Natl Vital Stat Rep. 2010;58(21): 1-40. Available at: http://www.cdc.gov/nchs/data/nvsr/nvsr58/nvsr58_21. pdf. Accessed January 9, 2015.

25. Goldberg LD, Edwards NC, Fincher C, Doan QV, Al-Sabbagh A, Meletiche DM. Comparing the cost-effectiveness of disease-modifying drugs for the first-line treatment of relapsing-remitting multiple sclerosis. J Manag Care Pharm. 2009;15(7):543-55. Available at: http://www.amcp.org/data/ jmcp/543-555.pdf. 
26. Xie J, Namjoshi M, Wu EQ, et al. Economic evaluation of denosumab compared with zoledronic acid in hormone-refractory prostate cancer patients with bone metastases. J Manag Care Pharm. 2011;17(8):621-43. Available at: http://www.amcp.org/WorkArea/DownloadAsset. aspx?id=12806

27. Truven Health Analytics. RED BOOK Online. Available at: http://www. redbook.com/redbook/online/. Accessed January 9, 2015.

28. Centers for Medicare \& Medicaid Services. Clinical laboratory fee schedule. 2014; Physician fee schedule look-up tool. 2013. Available at: http:// www.cms.hhs.gov/ClinicalLabFeeSched/02_clinlab.asp and http://www cms.hhs.gov/Pfslookup/. Accessed January 9, 2015.

29. U.S. Bureau of Labor Statistics. Consumer Price Index. Top picks. Consumer Price Index-all urban consumers. Available at: http://data.bls. gov/cgi-bin/surveymost?cu. Accessed January 14, 2015.

30. HCUPnet (Healthcare Cost and Utilization Project). HCP-3 nationwide inpatient sample for 2011 from principal diagnoses. Agency for Healthcare Research and Quality. Rockville, MD. Available at: http://www.hcup-us. ahrq.gov/nisoverview.jsp. Accessed January 14, 2015.
31. Sun Y, Friedman B. Tools for more accurate inpatient cost estimates with HCUP databases, 2009. Errata added October 25, 2012. HCUP Methods Series Report \#2011-04. Agency for Healthcare Research and Quality. Available at: http://www.hcup-us.ahrq.gov/reports/methods/2011_04.pdf. Accessed January 9, 2015.

32. Lee SJ, Hirsch JD, Terkeltaub R, et al. Perceptions of disease and healthrelated quality of life among patients with gout. Rheumatology (Oxford). 2009;48(5):582-86.

33. Sikirica V, Haim Erder M, Xie J, et al. Cost effectiveness of guanfacine extended release as an adjunctive therapy to a stimulant compared with stimulant monotherapy for the treatment of attention-deficit hyperactivity disorder in children and adolescents. Pharmacoeconomics. 2012;30(8):el-e15.

34. Kleinman NL, Brook RA, Patel PA, et al. The impact of gout on work absence and productivity. Value Health. 2007;10(4):231-37. 